\title{
Sildenafil no tratamento da hipertensão pulmonar após cirurgia cardíaca
}

\author{
Sildenafil for pulmonary hypertension treatment after cardiac surgery \\ Maria Regina Bentlin1, Adriana Saito², Ana Karina C. De Luca², Grasiela Bossolan², \\ Rossano C. Bonatto ${ }^{3}$, Antonio S. Martins ${ }^{4}$, Ligia M. S. S. Rugolo ${ }^{1}$
}

\section{Resumo}

Objetivo: Relatar o uso do Sildenafil no tratamento da hipertensão pulmonar em recém-nascido após cirurgia cardíaca.

Descrição: Recém-nascido de termo, feminino, com diagnóstico de dupla via de saída de ventrículo direito, hipoplasia de pulmonar e comunicação interventricular subaórtica, foi submetido à cirurgia de Blalock na primeira semana de vida. No pós-operatório, evoluiu com hipertensão pulmonar e hipoxemia persistente, não-responsiva ao óxido nítrico, porém com melhora da oxigenação após infusão endovenosa contínua de prostaglandina $\mathrm{E} 1$. Depois de várias tentativas malsucedidas de retirada da prostaglandina E1, optou-se pela introdução do Sildenafil via oral. Houve queda da resistência vascular pulmonar, com conseqüente melhora na oxigenação e, 48 horas após, foi possível suspender a infusão de prostaglandina E1.

Comentários: $O$ Sildenafil pode ser alternativa terapêutica na hipertensão pulmonar, especialmente quando não houver resposta à terapia convencional.

J Pediatr (Rio J). 2005;81(2):175-8: Sildenafil, hipertensão pulmonar, prostaglandina.

\section{Introdução}

A hipertensão pulmonar, doença de alta morbimortalidade na infância, tem etiologia multifatorial e ocorre em aproximadamente 1,9 nascido vivo a cada 1.000 . Caracteriza-se por aumento da resistência vascular pulmonar, resultando em shunt da direita para a esquerda, com

1. Doutora. Professora assistente da Disciplina de Neonatologia, Departamento de Pediatria, Faculdade de Medicina de Botucatu, Universidade Estadual Paulista (UNESP), Botucatu, SP

2. Médica, UTI Neonatal, Hospital das Clínicas, Faculdade de Medicina de Botucatu, UNESP, Botucatu, SP.

3. Doutor. Professor assistente, Disciplina de Cardiologia Infantil, Departamento de Pediatria, Faculdade de Medicina de Botucatu, UNESP, Botucatu, SP.

4. Doutor. Professor assistente da Disciplina de Cirurgia Cardíaca, Departamento de Cirurgia, Faculdade de Medicina de Botucatu, UNESP, Botucatu, SP.

Artigo submetido em 09.11.04, aceito em 17.01.05.

Como citar este artigo: Bentlin MR, Saito A, De Luca AK, Bossolan G, Bonatto RC, Martins AS, et al. Sildenafil no tratamento da hipertensão pulmonar após cirurgia cardíaca. J Pediatr (Rio J). 2005;81:175-8.

\section{Abstract}

Objective: To report on the use of sildenafil for pulmonary hypertension treatment of a newborn patient after cardiac surgery.

Description: A female, full term newborn infant with diagnosis of double outlet right ventricle, pulmonary hypoplasia and subaortic ventricular septal defect, was submitted to Blalock surgery in the first week of life. In postoperative the newborn had pulmonary hypertension and persistent hypoxia, without response to nitric oxide, but with improved oxygenation after continuous intravenous infusion of prostaglandin E1. After several failed attempts to discontinue prostaglandin E1, oral sildenafil was used. There was a decrease in pulmonary vascular resistance with consequent oxygenation improvement and 48 hours later it was possible to discontinue prostaglandin E1 infusion.

Comments: Sildenafil can be an alternative therapy for pulmonary hypertension, especially when there is no response to conventional therapy.

J Pediatr (Rio J). 2005;81(2):175-8: Sildenafil, pulmonary hypertension, prostaglandin.

passagem de sangue não-oxigenado pelo ducto arterioso ou forame oval ${ }^{1}$.

O tratamento objetiva a estabilização cardiovascular, a melhora da oxigenação e a redução da pressão na artéria pulmonar. No tratamento ${ }^{1-3}$, têm sido utilizados oxigênio, antagonistas de canais de cálcio, prostaciclinas e análogos, antagonistas dos receptores da endotelina e vasodilatadores, como óxido nítrico, mas, apesar de todo avanço terapêutico, apenas $50 \%$ dos pacientes apresentam resposta clínica ${ }^{1}$. Assim, têm sido investigadas novas opções terapêuticas, incluindo inibidores da enzima 5fosfodiesterase como o sildenafil, que promove aumento da concentração de guanosina monofosfato cíclico (GMPc) e conseqüente vasodilatação pulmonar ${ }^{4,5}$.

\section{Descrição do caso}

Trata-se de recém-nascido ( $R N)$ de termo, feminino, pesando $2.550 \mathrm{~g}$, com diagnóstico por ecocardiograma e 
cateterismo cardíaco de dupla via de saída de ventrículo direito, hipoplasia de tronco pulmonar e comunicação interventricular (CIV) subaórtica. Foi submetido à cirurgia de Blalock na primeira semana de vida. No pós-operatório, evoluiu com hipertensão pulmonar, diagnosticada por cateterismo cardíaco com medida de $76 \mathrm{mmHg}$, e hipoxemia persistente sem outra causa pulmonar que justificasse 0 quadro. Mantinha saturação de $70 \%$, não-responsiva à terapêutica, incluindo ventilação mecânica convencional e utilização de óxido nítrico (20 ppm). No $18^{\circ}$ dia de vida (110 do pós-operatório), após o início da infusão endovenosa (EV) contínua de prostaglandina E1 (PGE1), houve melhora da oxigenação. Com 50 dias de vida, após várias tentativas malsucedidas de retirada da PGE1, e da obtenção do consentimento por escrito dos pais, optou-se pelo uso de Sildefanil, na dose de $0,5 \mathrm{mg} / \mathrm{kg}$ via oral, a cada 6 horas. A pressão na artéria pulmonar, estimada por ecocardiograma com Doppler 4 horas antes da primeira dose de Sildenafil, era de $70 \mathrm{mmHg}$ e caiu para $55 \mathrm{mmHg}$ após 24 horas do início da medicação. Ao final da primeira semana de tratamento, a pressão pulmonar encontrava-se em $38 \mathrm{mmHg}$. Os efeitos na oxigenação foram observados nas primeiras 24 horas do uso e, depois de 48 horas, foi possível suspender a infusão de PGE1, com manutenção da saturação de oxigênio em torno de $90 \%$ em ar ambiente. Não houve hipotensão nem outros efeitos colaterais. A pressão arterial foi monitorizada por medida não-invasiva de hora em hora nas primeiras 24 horas do uso de Sildenafil, e posteriormente de forma periódica, antes e após a administração da droga. A criança recebeu alta hospitalar 15 dias depois com uso de Sildenafil, que foi mantido por mais 30 dias, com retirada gradual, sem intercorrências.
A evolução dos parâmetros ventilatórios e cardiovasculares com as terapêuticas instituídas encontram-se na Tabela 1.

\section{Discussão}

A ausência de resposta à terapia convencional e a necessidade de manutenção da droga de infusão EV contínua, de alto custo e não-isenta de efeitos colaterais, motivou-nos a buscar novas alternativas terapêuticas para o paciente. Estudos e relatos da utilização do Sildenafil via oral nos pareceram promissores e foram determinantes para sua utilização6-9.

O Sildenafil foi estudado em modelos animais de hipertensão pulmonar e mostrou-se vasodilatador pulmonar seletivo sem efeitos na pressão arterial sistêmica, potencializando os efeitos do óxido nítrico inalatório (ONi) quando administrado via oral ou EV5,10. É potente inibidor da enzima 5-fosfodiesterase que é responsável pela conversão do GMPc em GMP. Com a inibição dessa enzima, ocorre aumento da concentração de GMPc nos pulmões, com conseqüente relaxamento do músculo liso da parede vascular (Figura 1) ${ }^{6}$.

Esse medicamento é disponível para uso via oral, tendo boa absorção no trato gastrointestinal e início de ação 15 minutos após administração. Apresenta meia vida de 4 horas, e a eliminação é hepática. Os efeitos colaterais incluem cefaléia, rubor, tontura, dispepsia, congestão nasal e alterações visuais. A dose utilizada para vasodilatação pulmonar em adultos corresponde a $25-50 \%$ da dose recomendada em disfunção erétil. Para crianças, a dose sugerida é de 1 a $2 \mathrm{mg} / \mathrm{kg} / \mathrm{dia}^{6,11}$.

Tabela 1 - Dados ventilatórios e cardiovasculares do paciente

\begin{tabular}{|c|c|c|c|c|c|c|}
\hline Parâmetros & $\begin{array}{c}\text { PO } \\
\text { imediato }\end{array}$ & $\begin{array}{c}\text { Óxido } \\
\text { nítrico } *\end{array}$ & PGE1 † & $\begin{array}{c}\text { PGE1 }{ }^{\text {P }} \\
\text { pré-Sildenafil }\end{array}$ & $\begin{array}{c}\text { PGE1 }{ }^{\ddagger}+ \\
\text { Sildenafil § } \\
24 \text { horas após }\end{array}$ & Sildenafil § \\
\hline Dias de vida & 7 & 15 & 18 & 50 & 51 & 58 \\
\hline $\mathrm{FiO}_{2}$ & 1,0 & 1,0 & 0,6 & 0,5 & 0,4 & 0,21 \\
\hline Sat $\mathrm{O}_{2}(\%)$ & 60 & 70 & 95 & $96 \%$ & 96 & 90 \\
\hline $\mathrm{PaO}_{2}(\mathrm{mmHg})$ & 26 & 37 & 70 & 81 & 75 & 60 \\
\hline MAP & 12 & 12 & - & - & - & - \\
\hline PAM $(\mathrm{mmHg})$ & 48 & 55 & 80 & 78 & 75 & 65 \\
\hline $\mathrm{PAP}(\mathrm{mmHg})$ & 70 & 76 & 70 & 70 & 55 & 38 \\
\hline $\mathrm{D}(\mathrm{A}-\mathrm{a}) \mathrm{O}_{2}$ & 591 & 580 & 286 & 210 & 151 & 37 \\
\hline
\end{tabular}

$\mathrm{PO}=$ pós-operatório; $\mathrm{FiO}_{2}$ = fração inspirada de oxigênio; Sat $\mathrm{O}_{2}=$ saturação de oxigênio (oximetria de pulso); $\mathrm{PaO}{ }_{2}=$ pressão parcial de oxigênio (gasometria arterial); MAP = pressão média de vias aéreas; PAM = pressão arterial média (monitorização nãoinvasiva); PAP = pressão artéria pulmonar (ecocardiograma com Doppler); $D(A-a) \mathrm{O}_{2}=$ diferença alvéolo arterial de oxigênio.

* $20 \mathrm{ppm}$, via inalatória

$\dagger 0,1 \mu \mathrm{g} / \mathrm{kg} / \mathrm{min}$, via endovenosa

‡ $0,05 \mu \mathrm{g} / \mathrm{kg} / \mathrm{min}$, via endovenosa

$\S 2 \mathrm{mg} / \mathrm{kg} / \mathrm{dia}$, via oral

cateterismo cardíaco 


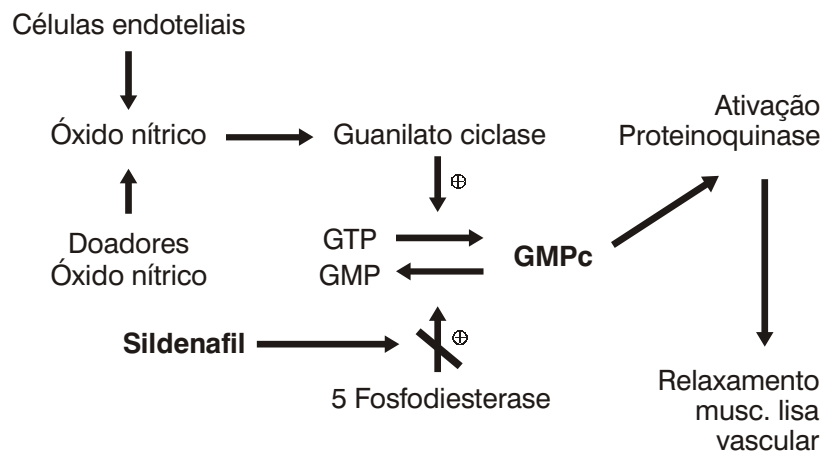

Figura 1 - Algoritmo do mecanismo de ação do Sildenafil, modificado de Abrams et al. ${ }^{6}$

Estudos realizados em adultos com hipertensão pulmonar mostram redução na resistência vascular pulmonar com o uso de Sildenafil na forma de monoterapia ou em associação com prostaciclina ${ }^{12}$ ou $\mathrm{ONi}^{13}$.

Os efeitos do Sildenafil na vasculatura pulmonar independem da etiologia da hipertensão pulmonar, que pode variar de acordo com a faixa etária. No período neonatal, a hipertensão pulmonar pode ser decorrente de aspiração de mecônio, sepse, pneumonia, asfixia perinatal, hérnia diafragmática congênita, hipoplasia pulmonar; na infância, em geral associa-se a doenças pulmonares ou seqüelas de pneumonite intersticial ${ }^{1}$. A hipertensão secundária à cardiopatia congênita ocorre independente da faixa etária.

O uso de Sildenafil em crianças limita-se a relatos de $\operatorname{casos}^{6-9}$ e apenas a um estudo randomizado que compara o ONi e Sildenafil intravenoso ${ }^{14}$.

Na hipertensão pulmonar, após cirurgia cardíaca, há relatos de três crianças, com idades de um dia, 6 semanas e 4 meses, que utilizaram Sildenafil via oral $(1 \mathrm{mg})$ em associação com ONi, após várias tentativas malsucedidas de retirada do óxido nítrico. Com a introdução do sildenafil, houve diminuição da pressão na artéria pulmonar, sem alteração na pressão sistêmica, sendo possível suspender o $\mathrm{ONi}^{7}$. Estudo randomizado envolvendo $15 \mathrm{RN}$ submetidos à cirurgia cardíaca para correção de defeitos dos septos ventricular e atrioventricular avaliou o uso do ONi e Ssildenafil intravenoso: sete RN receberam inicialmente ONi (20 $\mathrm{ppm})$ e depois Sildenafil $(0,35 \mathrm{mg} / \mathrm{kg})$ e $8 \mathrm{RN}$ receberam Sildenafil e depois ONi. A despeito do efeito vasodilatador pulmonar, potencializando o ONi em ambos os grupos, o Sildenafil reduziu a pressão sangüínea sistêmica, levando à hipotensão sistêmica e à piora da oxigenação, provavelmente por aumento do shunt intrapulmonar. Embora com algumas limitações metodológicas, o estudo alerta para a necessidade de novos estudos, pois foram encontrados efeitos indesejáveis como hipotensão, até então não-descritos $^{14}$.

Na hipertensão primária, há relato do uso de Sildenafil em criança de 4 anos que não respondeu ao óxido nítrico, mas que apresentou diminuição da pressão na artéria pulmonar com prostaciclina. Foi indicado Sildenafil, na dose $2 \mathrm{mg} / \mathrm{kg} / \mathrm{dia}$, via oral, a cada 6 horas, para reduzir os efeitos do uso a longo prazo da prostaciclina, entre eles as alterações ósseas como hiperostose, proliferação cortical e periostite. A prostaciclina foi retirada com sucesso ${ }^{6}$.

Erickson et al. ${ }^{8}$ utilizaram sildenafil via oral em 24 crianças, dos quais cinco eram RN, todos com hipertensão pulmonar refratária, após a retirada gradual do ONi. O Sildenafil permitiu a retirada do ONi em todos os pacientes, sem instabilidade dos mesmos. Recentemente, Carrol \& Dhillon ${ }^{9}$ relataram o uso de Ssildenafil em três crianças, sendo uma no pós-operatório de cirurgia cardíaca com boa resposta e duas com hipertensão pulmonar secundária à pneumonite intersticial. Uma delas não respondeu ao tratamento, fato esse atribuído à gravidade do quadro e uso tardio da droga.

Em nosso caso, o óxido nítrico não se mostrou eficaz no tratamento da hipertensão pulmonar. O ONi é o mais importante vasodilatador com efeito pulmonar seletivo, melhorando o prognóstico de pacientes hipoxêmicos e reduzindo a mortalidade e a necessidade de oxigenação por membrana extra-corpórea (ECMO). Entretanto, aproximadamente $50 \%$ dos pacientes não respondem a essa terapêutica, seja por apresentarem doenças graves de parênquima pulmonar, seja por disfunção miocárdica ou alterações na relação óxido nítrico-GMPc ${ }^{11}$. A disfunção miocárdica já existente em nosso paciente poderia justificar a nãoresposta ao óxido nítrico, mas é interessante observar que houve resposta à infusão contínua de PGE1.

Prostaciclinas e PGE1 e I2 são importantes vasodilatadores pulmonares em condições de hipóxia, possuindo efeitos vasoativos na circulação pulmonar fetal e neonatal e efeito sinérgico com outras drogas como os inibidores de fosfodiesterase 2,15 . A prostaciclina apresenta meia vida mais curta do que a PGE1, em torno de 1 a 2 minutos, tem menor efeito na ativação plaquetária e reduz a vasoconstricção pulmonar ${ }^{15}$.

A boa resposta em nosso paciente sugere que o Sildenafil possa ter papel no tratamento da hipertensão pulmonar após cirurgia cardíaca, situando-se atualmente como tratamento de resgate para aqueles pacientes que não responderam à terapêutica convencional. A administração via oral é outro aspecto a ser considerado, pois evita complicações a longo prazo do uso de prostaglandina/ prostaciclina e permite redução do tempo de internação.

Pela escassez de dados na literatura e por ser este um medicamento novo, ainda em fase experimental e de uso excepcional como resgate na hipertensão pulmonar grave em seres humanos, principalmente em RN, a descrição desse caso espera contribuir como nova experiência em que - Sildenafil foi benéfico como vasodilatador pulmonar, propiciando a retirada da PGE1, sem causar efeitos adversos a curto prazo no paciente.

O Sildenafil parece ser um dos agentes promissores no tratamento da hipertensão pulmonar, porém várias questões precisam ser mais bem esclarecidas antes que se possa recomendar o uso dessa medicação; entre elas se encon- 
tram a interação da enzima 5-fosfodiesterase e sua distribuição em tecidos extrapulmonares, os efeitos do Sildenafil na função miocárdica e em trocas gasosas pulmonares, o efeito hemodinâmico sistêmico na presença de sepse e o efeito em pacientes com disfunção hepática.

Os dados até então disponíveis apontam para a necessidade de estudos clínicos randomizados para avaliar a eficácia e a segurança desse medicamento no tratamento da hipertensão pulmonar; da mesma forma, são necessários estudos de farmacocinética para determinar a dose e a via de administração seguras em RN e crianças.

\section{Referências}

1. Suguihara C. Tratamento da hipertensão pulmonar persistente do recém-nascido. J Pediatr (Rio J). 2001;77 Suppl 1:S17-24.

2. Weinberger $B$, Weiss $K$, Heck DE, Laskin DL, Laskin JD. Pharmacologic therapy of persistent pulmonary hypertension of the newborn. Pharmacol Ther. 2001;89:67-79.

3. Crespo-Martinez C, Morales LV, Alonso RH, Alonso OB, Molero GR. Primary pulmonary hypertension and its management. Farm Hosp. 2004;28:48-55.

4. Zhao L, Mason NA, Morrell NW, Kojonazarov B, Sadykov A, Maripov $A$, et al. Sildenafil inhibits hypoxia-induced pulmonary hypertension. Circulation. 2001;04:424-8.

5. Shekerdemian LS, Ravn HB, Penny D. Intravenous Sildenafil lowers pulmonary vascular resistance in a model of neonatal pulmonary hypertension. Am J Resp Crit Care Med. 2002;165: 1098-102.

6. Abrams D, Schulze-Neick I, Magee AG. Sildenafil as a selective pulmonary vasodilator in childhood primary pulmonary hypertension. Heart. 2000;84:e4.

7. Atz AM, Wessel DL. Sildenafil ameliorates effects of inhaled nitric oxide withdrawal. Anesthesiology. 1999;91:307-10.
8. Erickson S, Reyes J, Bohn D, Adatia I. Sildenafil (Viagra) in childhood and neonatal pulmonary hypertension. J Am Coll Cardiol. 2002;39 Suppl:S402.

9. Carroll WD, Dhillon R. Sildenafil as a treatment for pulmonary hypertension. Arch Dis Child. 2003;88:827-8.

10. Weimann J, Ullrich R, Hromi J, Fujino Y, Clark MW, Bloch KD, et al. Sildenafil is a pulmonary vasodilator in awake lambs with acute pulmonary hypertension. Anesthesiology. 2000;92: 1702-12.

11. Travardi JN, Patole SK. Phosphodiesterase inhibitors for persistent pulmonary hypertension of the newborn: a review. Pediatr Pulmonol. 2003;36:529-35.

12. Ghofrani HA, Wiedeman R, Rose F, Olschewski H, Chermuly RT, Weissmann $\mathrm{N}$, et al. Combination therapy with oral sildenafil and inhaled iloprost for severe pulmonary hypertension. Ann Intern Med. 2002;136:515-22.

13. Michelakis E, Tymchak W, Lien D, Webster L, Hashimoto K, Archer $S$. Oral sildenafil is an effective and specific pulmonary vasodilator in patients with pulmonary arterial hypertension. Comparison with inhaled nitric oxide. Circulation. 2002;105:2398-403.

14. Stocker C, Penny DJ, Brizard CP, Cochrane AD, Soto $R_{,}$ Shekerdemian LS. Intravenous sildenafil and inhaled nitric oxide: a randomized trial in infants after cardiac surgery. Intensive Care Med. 2003;29:1996-2003.

15. Long WA, Rubin LJ. Prostacyclin and PGE1 treatment of pulmonary hypertension. Am Rev Respir Dis. 1987;136:773-6.

Correspondência:

Maria Regina Bentlin

Dep. de Pediatria, Faculdade de Medicina de Botucatu - UNESP

Distrito de Rubião Júnior

CEP 18618-000 - Botucatu, SP

Tel./Fax: (14) 3811.6274

E-mail: mbentlin@fmb.unesp.br 